\title{
ESTUDO DA DECOMPOSIÇÃO TÉRMICA DA MADEIRA DE OITO ESPÉCIES DA REGIÃO DO SERIDÓ, RIO GRANDE DO NORTE ${ }^{1}$
}

\author{
Angélica de Cássia Oliveira Carneiro² ${ }^{2}$ Rosimeire Cavalcante dos Santos ${ }^{3}$, Renato Vinícius Oliveira \\ Castro $^{4}$, Ana Flávia Neves Mendes Castro ${ }^{5}$, Alexandre Santos Pimenta ${ }^{3}$, Edna Moura Pinto ${ }^{3}$ e Isabel \\ Cristina Nogueira Alves
}

\begin{abstract}
RESUMO - Este trabalho teve como objetivo avaliar o perfil de decomposição térmica da madeira de oito espécies utilizando as técnicas de termogravimetria (TGA) e calorimetria diferencial exploratória (DSC), bem como a consistência desses métodos para decidir quais espécies devem ser indicadas para produção de energia e carvão vegetal, comparando-os com os rendimentos gravimétricos da carbonização obtidos a partir de carbonizações em mufla de laboratório. Para as análises por TGA e DSC, amostras de serragem foram aquecidas à taxa de $10{ }^{\circ} \mathrm{C} \mathrm{min}{ }^{-1}$, sob atmosfera de nitrogênio, em vazão de $50 \mathrm{ml} \mathrm{min}^{-1}$ até a temperatura final de $600{ }^{\circ} \mathrm{C}$. Foram determinados os valores de densidade básica e poder calorífico superior da madeira. As carbonizações foram realizadas em mufla de laboratório à taxa de $50{ }^{\circ} \mathrm{C} \mathrm{h}^{-1}$ até a temperatura máxima de $450{ }^{\circ} \mathrm{C}$, obtendo-se os rendimentos em carvão vegetal. Também foram determinados os valores de densidade aparente do carvão das oito espécies após a carbonização. Os resultados das análises térmicas indicaram perfis de decomposição pirolítica das madeiras e evidenciaram aquelas mais estáveis à ação térmica. O comportamento da estabilidade térmica das madeiras das oito espécies foi confirmado pela análise dos rendimentos da carbonização em mufla, em comparação com a análise por TGA e DSC. As madeiras de Aspidosperma pyrifolium e Mimosa tenuiflora apresentaram maior estabilidade térmica e, portanto, maiores rendimentos em carvão vegetal, bem como maiores densidades aparentes. As técnicas de termogravimetria e calorimetria diferencial exploratória são satisfatórias para avaliação da decomposição térmica das madeiras e garantem consistência na escolha de madeiras que resultam em maior rendimento e maior qualidade do carvão vegetal.
\end{abstract}

Palavras-chave: Análise termogravimétrica; Calorimetria diferencial exploratória; Estabilidade térmica da madeira.

\section{STUDY OF THE THERMAL DECOMPOSITION OF EIGHT WOOD SPECIES FROM SERIDÓ REGION, RIO GRANDE DO NORTE}

\begin{abstract}
The objective of this study was to evaluate the profile of the thermal decomposition of the wood of eight species using thermogravimetry (TGA) techniques and differential scanning calorimetry (DSC) as well as the consistency of these methods to decide which species should be listed for production of energy and charcoal, comparing them with the carbonization gravimetric yield obtained from carbonization in laboratory muffle. For analysis by DSC and TGA, sawdust samples were heated at a rate of $10^{\circ} \mathrm{C} \mathrm{min}^{-1}$ in nitrogen atmosphere, at a flow rate of $50 \mathrm{ml} \mathrm{min}-1$ until final temperature of $600^{\circ} \mathrm{C}$. The values of basic density and higher calorific value of the wood were determined. The carbonizations were conducted in laboratory muffle at a rate of $50{ }^{\circ} \mathrm{C}_{\text {hour }}^{-1}$ to a maximum temperature of $450^{\circ} \mathrm{C}$ to give the charcoal yields. The values of coal apparent
\end{abstract}

\footnotetext{
${ }^{1}$ Recebido em 10.12.2012 aceito para publicação em 15.10.2013.

${ }^{2}$ Departamento de Engenharia Florestal, Universidade Federal de Viçosa, UFV, Brasil. E-mail:<cassiacarneiro1@gmail.com>.

${ }^{3}$ Universidade Federal do Rio Grande do Norte, UFRN, Brasil. E-mail:<meire_caico@yahoo.com.br>, <alexandre_spimenta@hotmail.com>e <emourapinto@yahoo.com.br>.

${ }^{4}$ Departamento de Engenharia Florestal da Universidade de Brasília, UnB. E-mail:<castrorvo@ymail.com>.

${ }^{5}$ Programa de Pós-graduação em em Ciência Florestal na Universidade Federal de Viçosa, UFV, Brasil. E-mail: $<$ mendesafn@hotmail.com>.

${ }^{6}$ Departamento de Engenharia Florestal da Universidade Federal Rural do Rio de Janeiro. E-mail:<isabel.alves@posgrad.ufla.br>.
} 
density from the eight species after carbonization were also determined. The results of the thermal analysis showed the profiles of pyrolytic decompoistion of the wood and evidenced those more stable to thermal action. The behavior of thermal stability of the wood of the eight species was confirmed by analysis of the yields of carbonization in muffle, compared to analysis by DSC and TGA. Woods of Aspidosperma pyrifolium and Mimosa tenuiflora showed higher thermal stability, thus higher yields in charcoal, as well as higher apparent densities. The thermogravimetry techniques and differential scanning calorimetry are satisfactory for evaluation of wood thermal decomposition and ensure consistency in the choice of woods that result in higher yields and higher quality charcoal.

Keywords: Differential scanning calorimetry; Thermogravimetric analysis; Wood thermal stability.

\section{INTRODUÇÃO}

Entre os estados brasileiros que utilizam a madeira como principal fonte de energia, o Estado do Rio Grande do Norte apresenta realidade florestal caracterizada pela elevada dependência da lenha e do carvão vegetal, tanto pelo setor domiciliar quanto pelos setores industrial e comercial. A região do Seridó, tem essa realidade agravada pelas suas condições edafoclimáticas (baixa intensidade de chuvas, concentradas em poucos meses do ano, e solos rasos) e pelo baixo nível tecnológico de suas indústrias (SEPLAN, 2004).

No Estado do Rio Grande do Norte, a lenha apresenta preço inferior ao dos demais combustíveis, consolidando-se, assim, como a principal fonte de energia para alguns setores, com destaque para o setor da cerâmica vermelha. Se, por um lado, a lenha proporciona ao produto final valor mais competitivo, por outro lado o desmatamento sistemático já se constitui em ameaça de desertificação.

A dependência histórica do semiárido nordestino pelo corte da vegetação é perceptível, e a situação inspira cuidados. Segundo Giulietti et al. (2003), o uso da lenha é a mais importante contribuição do extrativismo no Nordeste registrada nos Censos do IBGE, já que estes não incluem o valor do pasto nativo. As cerâmicas, olarias, padarias e casas de farinha são usuárias tradicionais, e as indústrias de gesso e cimento, usuárias em expansão.

Nessa perspectiva, Schwob (2007), ao tratar do consumo da lenha na região do Seridó, RN, estima um montante na ordem de $60.000 \mathrm{~m}^{3}$. $\mathrm{mês}^{-1}$, que são consumidos somente pelo setor da cerâmica vermelha, incluindo também uso de resíduos, mesmo que numa pequena proporção, com uma relação específica de consumo de lenha da ordem de $1,8 \mathrm{~m}^{3}$. milheiro $^{-1}$ de produto (cerca de 1.000 kcal. $\mathrm{kg}^{-1}$ ).
Atualmente, o consumo energético mensal dessas cerâmicas na região do Seridó totaliza cerca de $106.000 \mathrm{~m}^{3}$ de lenha e 2.500.000 quilowatts de energia elétrica, segundo informações do sindicato da indústria cerâmica para construção do RN (daquele Estado) (SINDICER, 2012).

A obtenção de energia da madeira a partir do fornecimento de altas temperaturas promove a decomposição térmica dos seus componentes químicos, passando por um processo de carbonização sob atmosfera inerte e, ou, combustão sob atmosfera oxidante. No entanto, cada um desses componentes apresenta essa decomposição mais pronunciada em faixas de temperaturas distintas.

Segundo Conesa (1995), cada fração dos componentes da madeira tem uma cinética de decomposição térmica bem diferenciada. As hemiceluloses sofrem maiores picos de degradação entre 200 e $300{ }^{\circ} \mathrm{C}$, a celulose entre 240 e $350{ }^{\circ} \mathrm{C}$ e a lignina entre 350 e $500{ }^{\circ} \mathrm{C}$.

Devido à complexidade do processo de conversão da madeira em energia, faz-se necessário avaliar o perfil de sua decomposição térmica, principalmente considerando que a madeira é material heterogêneo por apresentar, entre outras características, diferenças significativas em suas propriedades, inclusive nas propriedades químicas de cada espécie e, até mesmo, dentro da mesma espécie.

Para isso é necessário utilizar técnicas que facilitem o entendimento do processo. Análises térmicas são convenientes e reprodutíveis e consideradas métodos úteis para caracterização de materiais orgânicos heterogêneos (OLIVEIRA, 2003).

As técnicas termoanalíticas têm sido definidas como métodos pelos quais se mede determinada propriedade física de uma amostra em função do tempo 
ou da temperatura; como um caso particular das técnicas termoanalíticas, a análise termogravimétrica (TGA) é a que acompanha a variação da propriedade física da massa da amostra em função do tempo (com a temperatura constante) ou da temperatura (CAVALHEIRO, 1995), o que permite acompanhar possíveis processos de degradação do material na temperatura estudada (OLIVEIRA, 2009).

Com esse método é possível determinar em qual temperatura o processo de degradação térmica se inicia e quando é mais acentuado, com base na massa da amostra, além de fornecer a quantidade de resíduo que é produzida em determinada temperatura, sendo, dessa maneira, ferramenta importante quando se desejam selecionar espécies para fins energéticos.

Paralelamente, é possível, por meio da calorimetria diferencial exploratória (DSC), medir a quantidade de calor absorvido ou liberado pela amostra, sendo essas alterações importantes porque apontam em quais temperaturas ocorrem mudanças físicas (como fusão ou vaporização) ou químicas (OLIVEIRA, 2009), ou seja, mede-se a variação de entalpia que ocorre entre a amostra e a referência durante o processo de aquecimento/resfriamento (BERNAL et al., 2002). Essa técnica permite, entre outras coisas, determinar a temperatura de fusão e de decomposição, além de fornecer dados sobre a estabilidade térmica de compostos (SILVA et al., 2004).

Diante do exposto, é importante estudar o perfil da estabilidade térmica da madeira quando se pretendem selecionar espécies que apresentam potencial energético utilizando como referência, além da estabilidade térmica, o rendimento gravimétrico, a densidade e o poder calorífico da madeira mais o rendimento e qualidade do carvão vegetal.

Como forma de ampliar a base de conhecimentos sobre as espécies de madeira que ocorrem em áreas sob manejo florestal na região do Seridó, no Rio Grande do Norte, visando à produção de energia, esta pesquisa teve como objetivo avaliar o perfil da decomposição térmica da madeira de oito espécies do semiárido nordestino utilizando as técnicas da análise termogravimétrica (TGA) e da calorimetria diferencial exploratória (DSC), como também analisar a densidade e o poder calorífico superior da madeira, além do rendimento gravimétrico em carvão vegetal, visando indicar as espécies de maior potencial energético.

\section{MATERIAL E MÉTODOS}

O experimento foi realizado utilizando-se madeiras provenientes de um povoamento com 20 anos de idade, localizado no Município de Caicó, Estado do Rio Grande do Norte, Brasil, situado entre as coordenadas geográficas 6 18' 55,3'’ S e 36 59' 28,8' ’ W. A área compreende um total de 2.006 ha, sendo 367,9 ha de vegetação nativa da caatinga submetida a manejo florestal. O produto madeireiro explorável refere-se à lenha com destino energético (lenha e carvão vegetal). O projeto implementado nessa área visa à exploração da Caatinga Arbustiva Arbórea Aberta, manejada em regime florestal sustentável, para produzir 19.404,6 estéreos (st) de lenha através da exploração da área manejada, com produtividade média de 52,8 estéreos por hectare (st ha-1), em ciclos de corte de 15 anos, utilizando-se corte seletivo da vegetação lenhosa em área não contínua.

As espécies foram selecionadas baseando-se em dados do inventário florestal do plano de manejo da área. Foram realizadas as coletas para o estudo da potencialidade energética das espécies, com base na média quadrática dos diâmetros das oito espécies que apresentaram maior Índice de Valor de Importância (IVI). As informações gerais sobre as espécies estudadas e os critérios estabelecidos para a condução da amostragem constam na Tabela 1.

A seleção das árvores-amostra foi feita excluindo-se aquelas que apresentavam defeitos. Foram coletadas quatro árvores por espécie, totalizando 32 exemplares. Dessas árvores foram retirados toretes com comprimento médio de 70 cm, entre o diâmetro a 1,3 m de altura (dap) e a base da árvore (cerca de $30 \mathrm{~cm}$ acima do nível do solo). Desses toretes foram retirados discos com 2,5 cm de espessura. Os discos foram subdivididos em quatro cunhas, sendo utilizadas duas cunhas opostas para determinação das análises termogravimétrica e calorimétrica diferencial, densidade básica, poder calorífico superior e as demais para carbonização. As análises foram realizadas com amostra composta representativa da árvore.

Os procedimentos utilizados para análise da densidade básica da madeira estão de acordo com o método de imersão em água, descrito por Vital (1984). Os resultados foram calculados como a média aritmética das quatro árvores por espécie, levando-se em consideração cinco pontos de amostragem ao longo do torete de cada árvore-amostra.

Revista Árvore, Viçosa-MG, v.37, n.6, p.1153-1163, 2013 
Tabela 1 - Espécies estudadas e critérios utilizados para amostragem experimental. Table 1 - Species studied and criteria used for experimental sampling.

\begin{tabular}{llrr}
\hline Nome científico & Nome Vulgar & $q(\mathrm{~cm})$ & IVI(\%) \\
\hline Mimosa tenuiflora (Willd.) Poiret & Jurema-preta & 28,4 & 45,75 \\
Aspidosperma pyrifolium Mart & Pereiro & 32,8 & 28,68 \\
Croton sonderianus Müll. Arg. & Marmeleiro & 16,6 & 6,32 \\
Caesalpinia pyramidalis Tul. var. pyramidalis & Catingueira & 31,2 & 2,36 \\
Bauhinia cheilantha (Bong.) Steud. & Mororó & 25,3 & 0,19 \\
Commiphora leptophloeos (Mart.) J.B. Gillett & Imburana & 27,1 & 1,70 \\
Piptadenia stipulacea (Benth.) Ducke & Jurema-branca & 21,4 & 1,21 \\
Combretum leprosum Mart. & Mofumbo & 16,5 & 0,11 \\
\hline
\end{tabular}

$q$ = diâmetro médio amostrado (cm); e IVI = índice de valor de importância da espécie (\%).

O poder calorífico superior das madeiras foram determinados de acordo com a metodologia descrita pela norma da ABNT NBR 8633 (ABNT, 1984), utilizando-se uma bomba calorimétrica adiabática. As amostras foram classificadas em peneiras de 40/ 60 mesh. As frações das amostras, retidas na peneira de 60 mesh, foram secas em estufa a $103 \pm 2{ }^{\circ} \mathrm{C}$ até peso constante, para determinação do poder calorífico superior.

As análises termogravimétricas foram realizadas utilizando o aparelho TGA-60H da SHIMADZU sob atmosfera de gás nitrogênio, a uma vazão constante de $50 \mathrm{ml} . \mathrm{min}^{-1}$, com o uso de amostras de serragem de $4 \mathrm{mg}$. A granulometria da serragem foi padronizada e passada em peneiras de 200 e de 270 mesh (ASTM, 1974), utilizando-se para as análises a fração retida nesta última. As análises foram conduzidas desde a temperatura ambiente até a temperatura máxima de $600{ }^{\circ} \mathrm{C}$, com taxa de aquecimento de $10{ }^{\circ} \mathrm{C}$. min ${ }^{-}$ ${ }^{1}$. Com base na massa inicial de cada amostra, foram calculadas as perdas de massa, expressas em porcentagem, subtraindo-se do valor da massa final obtida nos seguintes intervalos de temperatura: 25$100{ }^{\circ} \mathrm{C}, 100-200^{\circ} \mathrm{C}, 200-300{ }^{\circ} \mathrm{C}, 300-400{ }^{\circ} \mathrm{C}, 400-500$ ${ }^{\circ} \mathrm{C}$ e $500-600{ }^{\circ} \mathrm{C}$. Posteriormente, foram gerados termogramas, utilizando-se o programa estatístico disponível para o equipamento, e, a seguir, as funções obtidas foram interpoladas e derivadas. As funções interpoladas originaram as curvas termogravimétricas para análise do comportamento da resistência térmica da madeira com base na perda de massa sob as referidas faixas de temperatura, e as derivadas indicaram, por meio da análise térmica diferencial, as temperaturas nas quais ocorreram as maiores perdas de massa nos intervalos selecionados visualmente nos gráficos. Os valores da massa residual foram obtidos a partir do somatório dos valores das perdas de massa observados em cada intervalo, decrescido de 100 .

Para análise do DSC, foi utilizada a mesma fração granulométrica do TGA, ou seja, o material que passou pela peneira de 200 mesh e ficou retido na de 270 mesh. Para determinar os parâmetros cinéticos da madeira, as medidas termoanalíticas foram obtidas com um aparelho DSC 60 da marca SHIMADZU, sendo as amostras analisadas em cápsulas fechadas de alumínio. Utilizaram-se 2 mg de madeira sob um fluxo de nitrogênio de $50 \mathrm{ml} \cdot \mathrm{min}^{-1}$, e os termogramas foram obtidos a partir da temperatura ambiente até $550{ }^{\circ} \mathrm{C}$, com taxa de aquecimento de $10^{\circ} \mathrm{C} \cdot \mathrm{min}^{-1}$.

As carbonizações da madeira foram realizadas em forno mufla de laboratório com controle de temperatura. O controle do aquecimento foi conduzido à taxa de $50^{\circ} \mathrm{C} . \mathrm{h}^{-1}$, o que corresponde a uma taxa média de $1,07^{\circ} \mathrm{C} \cdot \min ^{1}$. A temperatura inicial foi sempre igual a $150^{\circ} \mathrm{C}$ e a temperatura máxima, de $450^{\circ} \mathrm{C}$, permanecendo estabilizada por um período de $60 \mathrm{~min}$. O tempo total de carbonização foi, portanto, de $7 \mathrm{~h}$. Foram realizadas três repetições por tratamento, utilizando-se em cada ensaio, aproximadamente, $250 \mathrm{~g}$ de madeira em forma de cunhas sob amostragem composta das árvoresamostra. As cunhas foram previamente secas em estufa a $105 \pm 3^{\circ} \mathrm{C}$ e, posteriormente, acondicionadas em um conteiner metálico com dimensões nominais de $30 \mathrm{~cm}$ de comprimento e $12 \mathrm{~cm}$ de diâmetro, o qual foi levado ao interior da mufla.

Após cada carbonização, foram determinados, com

Revista Árvore, Viçosa-MG, v.37, n.6, p.1153-1163, 2013 
base na massa de madeira seca, os rendimentos gravimétricos em carvão. A densidade relativa aparente do carvão foi determinada de acordo com o método proposto por Vital (1984), utilizando-se amostras de 5 g de carvão e mercúrio como fluido. Os resultados foram calculados como a média aritmética, levando em consideração os pontos de amostragem ao longo do torete de cada árvore-amostra.

Os dados foram obtidos considerando-se um delineamento inteiramente casualizado, com oito tratamentos (oito espécies) e quatro repetições (árvoresamostra), totalizando 32 unidades amostrais. Para verificar as pressuposições da análise de variância, os dados foram submetidos a teste de Lilliefors para testar a normalidade dos dados e ao teste de Cochran para avaliar a homogeneidade das variâncias. Em seguida, procedeu-se à análise de variância pelo teste $\mathrm{F}$, sendo as médias comparadas pelo teste de Tukey. Para as variáveis que não atenderam às pressuposições da análise de variância, foi aplicado o teste não paramétrico de Kruskall-Wallis. Em todas as análises estatísticas, considerou-se o nível de significância de 5\%. As análises estatísticas foram realizadas com o auxílio do Programa STATISTICA 8.0.

\section{RESULTADOS}

Na Tabela 2 estão apresentados os valores da densidade básica e o poder calorífico da madeira, rendimento gravimétrico e densidade aparente do carvão vegetal das oito espécies analisadas.

De maneira geral, espera-se obter, em qualquer carbonização, maior rendimento gravimétrico em carvão vegetal, uma vez que maiores valores dessa variável são desejáveis, pois resulta em maior massa de carvão vegetal e, consequentemente, maior produtividade dos fornos. Entretanto, o rendimento gravimétrico em carvão vegetal pode apresentar grande variação, pois é influenciado por diversas variáveis, tanto da matéria-prima quanto do processo de transformação (pirólise). Pode-se dizer que a taxa de aquecimento, a temperatura final e o tempo de carbonização são as principais variáveis que influenciam na quantidade de carvão vegetal obtido.

Analisando a Tabela 2, observa-se que o rendimento gravimétrico das oito espécies avaliadas variou de $32,8 \%$ na madeira de pereiro (Aspidosperma pyrifolium) até 40,7\% na madeira de jurema-preta (Mimosa tenuiflora).
Esses valores são semelhantes aos obtidos em madeiras de Eucalyptus sp., conforme pode ser observado na literatura, que variam aproximadamente de $26 \%$ a $41 \%$ (SANTOS, 2010; OLIVEIRA et al., 2010; CASTRO, 2011). Oliveira (2003), estudando três espécies do semiárido nordestino, que também foram avaliadas neste estudo, observou valores semelhantes aos obtidos neste estudo, sendo o rendimento gravimétrico em carvão de Mimosa tenuiflora de 39,70\%. Em Aspidosperma pyrifolium, esse rendimento foi de 34,96\% e em Croton sonderianus, $35,68 \%$, apesar de a marcha de carbonização utilizada ser diferente da empregada neste estudo. Portanto, vale ressaltar que, devido ao fato de o rendimento gravimétrico ser influenciado pelas variáveis do processo de produção do carvão vegetal, fica difícil estabelecer comparação entre os trabalhos.

Quanto à densidade aparente do carvão vegetal, pode-se dizer que é influenciada pela densidade da madeira que lhe deu origem, pela temperatura final e pelo tempo de carbonização, pois, quanto maior a exposição da madeira ao calor, maior a perda de massa em relação ao volume.

Analisando a Tabela 2, verifica-se ampla variação da densidade aparente do carvão vegetal, conforme foi observado na densidade básica da madeira na Tabela 1. Observa-se, ainda, que as madeiras que apresentaram maior densidade básica produziram carvão vegetal com maior densidade aparente, o que já era esperado, uma vez que altas densidades da madeira estão relacionadas com a produção de carvão com densidade aparente mais alta (BRITO; BARRICHELLO, 1977; OLIVEIRA, 1988).

Na Figura 1A são apresentados os termogramas referentes às análises por TGA das espécies estudadas, que correspondem à perda de peso, em porcentagem, em função da temperatura, enquanto na Figura 1B são mostradas as curvas de DSC das madeiras das oito espécies.

Os termogramas apresentados na Figura 1A indicam que a perda de massa, refletida pela degradação térmica das madeiras das oito espécies, ocorreram de forma similar. De maneira geral, observou-se que há, principalmente, duas etapas de perda de massa, sendo a primeira atribuída à eliminação da água (umidade da madeira) e a segunda, à degradação da madeira propriamente dita. Resultados semelhantes das diversas madeiras foram observados na literatura (RIEGEL et

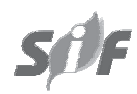

Revista Árvore, Viçosa-MG, v.37, n.6, p.1153-1163, 2013 
Tabela 2 - Densidade básica e poder calorífico da madeira, rendimento gravimétrico e densidade aparente do carvão vegetal das oito espécies analisadas.

Table 2 - Basic density and calorific value of the wood, gravimetric yield and apparent density of charcoal from eight species analyzed in the study.

\begin{tabular}{|c|c|c|c|c|}
\hline Espécie & $\begin{array}{l}\text { Densidadebásica } \\
\left(\mathrm{g} . \mathrm{cm}^{-3}\right)^{(*)}\end{array}$ & 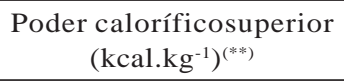 & $\operatorname{RGCV~}(\%)^{(* *)}$ & $\begin{array}{c}\text { Densidade aparente } \\
\left({\left.\mathrm{g} . \mathrm{cm}^{-3}\right)^{(* *)}}\right.\end{array}$ \\
\hline Mimosa tenuiflora & $0,90 \mathrm{~b}$ & 4823 a & $40,7 \mathrm{a}$ & 0,64 a \\
\hline Aspidosperma pyrifolium & $0,62 \mathrm{f}$ & $4720 \mathrm{~b}$ & $32,8 \mathrm{~b}$ & $0,48 \mathrm{~cd}$ \\
\hline Croton sonderianus & 0,66 e & 4646 bc & $33,8 \mathrm{~b}$ & $0,52 \mathrm{bc}$ \\
\hline Caesalpinia pyramidalis & $0,79 \mathrm{c}$ & $4442 \mathrm{f}$ & $36,4 \mathrm{ab}$ & $0,55 \mathrm{~b}$ \\
\hline Bauhinia cheilantha & 0,97 a & $4616 \mathrm{~cd}$ & 40,4 a & 0,62 а \\
\hline Commiphora leptophloeos & $0,29 \mathrm{~g}$ & 4479 ef & $37,0 \mathrm{ab}$ & $0,20 \mathrm{f}$ \\
\hline Piptadenia stipulacea & $0,76 \mathrm{~d}$ & 4557 de & $34,1 \mathrm{~b}$ & 0,44 de \\
\hline Combretum leprosum & 0,68 e & $4602 \mathrm{~cd}$ & $34,6 \mathrm{~b}$ & 0,40 e \\
\hline
\end{tabular}

Médias seguidas da mesma letra entre as espécies, em uma mesma variável, não diferem entre si, pelo teste de Kruskall-Wallis (*) ou de Tukey a 5\% de significância $(* *)$.

al., 2008; FRANCHESCHI et al., 2008; ELYOUNSSI et al., 2012).

Na Tabela 3, pode-se observar a perda de massa em relação à quantidade inicial de amostra em razão das faixas de temperatura estabelecidas, fornecidas pelo TGA, indicando a intensidade da decomposição térmica das espécies avaliadas. Com base na Figura 1A e na Tabela 3, verifica-se que, em todas as oito espécies, a maior perda de massa ocorreu na faixa de temperatura de 300 a $400{ }^{\circ} \mathrm{C}$, que, conforme Yang et al. (2007), é a faixa de temperatura em que se dá a maior degradação da celulose, que é o composto mais abundante da madeira.

Analisando a Figura 1B, observa-se que as curvas de DSC das oito espécies em estudo foram semelhantes, apresentando pico endotérmico na faixa de $80{ }^{\circ} \mathrm{C}$ a $100^{\circ} \mathrm{C}$, que pode ser atribuído à evaporação da água da madeira. A quantidade de energia consumida vai depender da quantidade de água contida no material, e a reação passa a ser exotérmica a aproximadamente $350^{\circ} \mathrm{C}$, coincidindo com os picos de máxima perda de massa, quando ocorre maior degradação da celulose, com liberação de voláteis.

A Tabela 4 apresenta as temperaturas em que ocorrem os picos de máximo e mínimo da curva de DSC e o início do processo exotérmico das oito espécies avaliadas, bem como a variação de entalpia $(\Delta \mathrm{H})$.

Observa-se na Tabela 4 que, nas oito espécies, a temperatura em que ocorre o pico mínimo da curva de DSC (pico endotérmico) não variou muito, sendo o menor valor $77,00^{\circ} \mathrm{C}$ e o maior, $82,09^{\circ} \mathrm{C}$. Jáno pico máximo da curva de DSC (pico exotérmico) a variação foi maior,

Revista Árvore, Viçosa-MG, v.37, n.6, p.1153-1163, 2013 sendo o menor valor $362,47^{\circ} \mathrm{C}$ e o maior, $392,96^{\circ} \mathrm{C}$. A temperatura de início da fase exotérmica ficou próxima a $320^{\circ} \mathrm{C}$, e a partir dessa temperatura acontece maior degradação da celulose (YANG et al., 2007).

\section{DISCUSSÃO}

Conforme mostrado na Tabela 2, houve ampla variação da densidade básica nas oito espécies de madeira avaliadas, sendo o menor valor 0,29 g.cm $\mathrm{cm}^{-3}$ na madeira de imburana (Commiphora leptophloeos) e, o maior, 0,97 g. $\mathrm{cm}^{-3}$ na madeira de mororó (Bauhinia cheilantha). Os valores evidenciam a variabilidade dessa característica nas madeiras que são comercializadas nessa área, o que implica tanto quantidade de energia armazenada por unidade de volume quanto custos com seu transporte.

Melo et al. (2006) estudaram a variação da densidade em madeiras do semiárido nordestino, no Estado da Paraíba, quando observaram nas madeiras de Mimosa tenuiflora e Croton sonderianus os seguintes valores, respectivamente: 0,99 g. $\mathrm{cm}^{-3} \mathrm{e}$ 0,68 g.cm ${ }^{-3}$. Araújo et al. (2004), estudando um povoamento de Mimosa tenuiflora, encontraram valores médios de 0,77 g.cm ${ }^{-3}$. Melo et al. (2007), ao avaliarem a densidade básica da madeira de Aspidosperma pyrifolium, constataram valores médios equivalentes a 0,79 g. $\mathrm{cm}^{-3}$. Estudando a madeira de Commiphora leptophloeos, Carvalho (2009) observou valor médio de densidade básica de $0,43 \mathrm{~g} \cdot \mathrm{cm}^{-3}$, sendo superior ao deste estudo.

Os valores de poder calorífico obtidos nas 
(A)

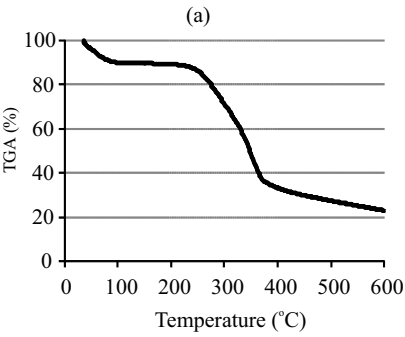

(c)

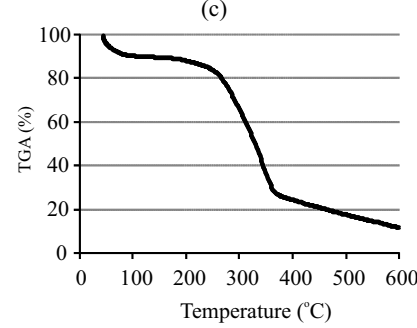

(e)

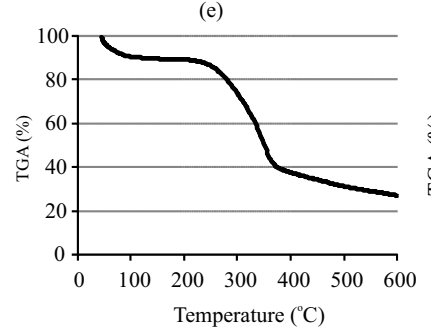

(g)

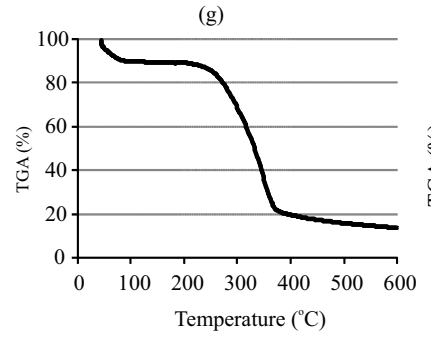

(b)

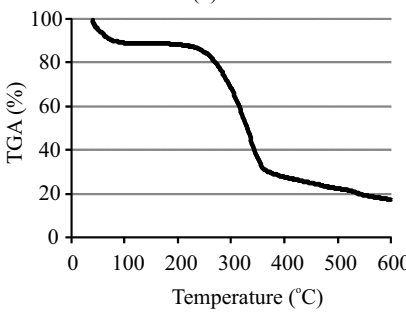

(d)
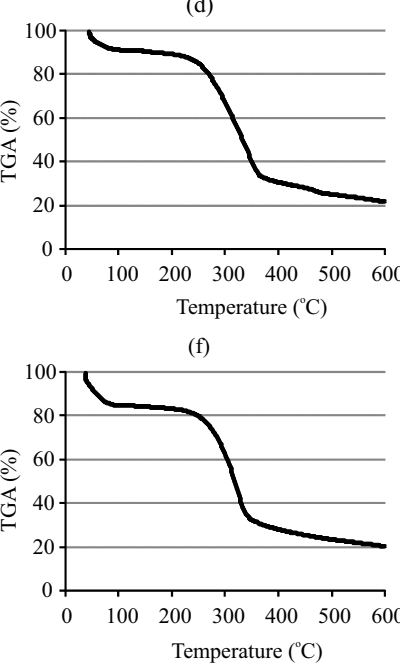

(h)

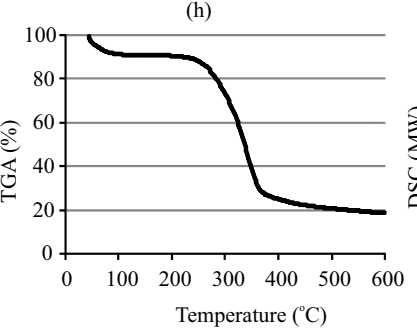

(a)

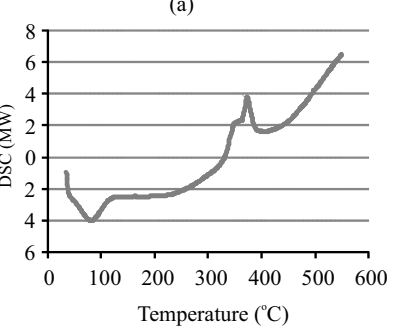

(c)

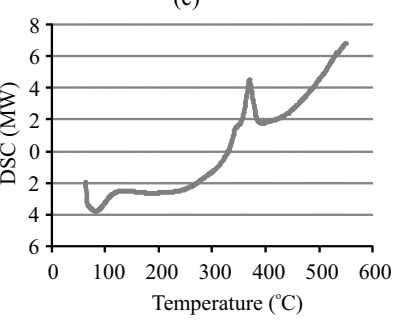

(e)

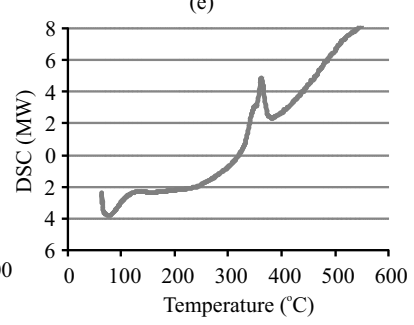

(g)

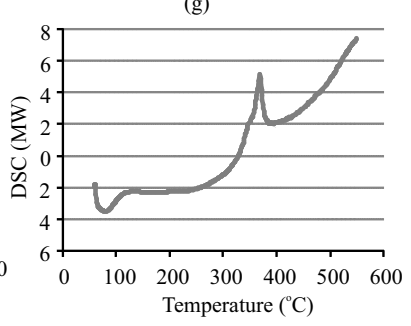

(B)

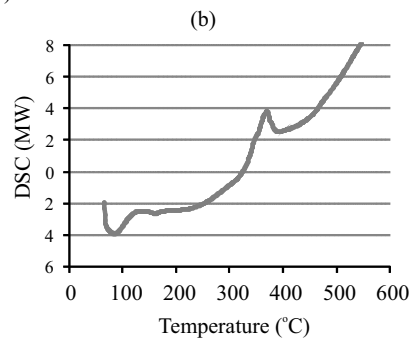

(d)

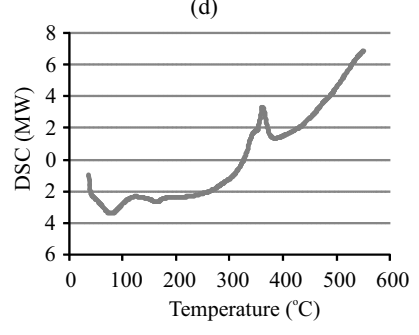

(f)

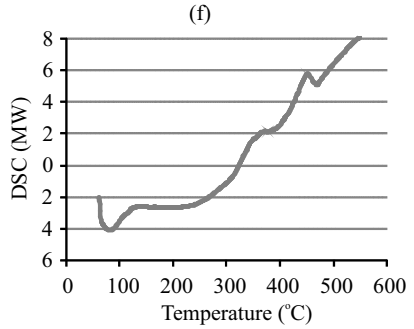

(h)

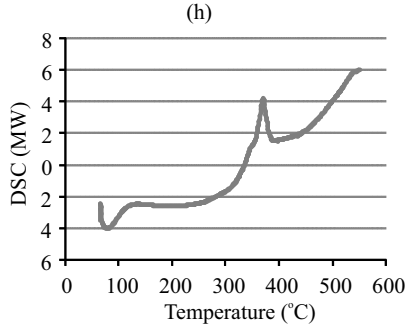

Figura 1 - Termogramas obtidos por TGA (A) e curvas de DSC (B) da madeira de Mimosa tenuiflora (a); Aspidosperma pyrifolium (b); Croton sonderianus (c); Caesalpinia pyramidalis (d); Bauhinia cheilantha (e); Commiphora leptophloeos (f); Piptadenia stipulacea (g); e Combretum leprosum (h).

Figure 1 - Thermograms obtained by TGA (A) and DSC curves (B) of Mimosa tenuiflora wood (a); Aspidosperma pyrifolium (b); Croton sonderianus (c); Caesalpinia pyramidalis (d); Bauhinia cheilantha (e); Commiphora leptophloeos (f); Piptadenia stipulacea ( $g$ ); and Combretum leprosum ( $h$ ).

madeiras avaliadas estão próximos ao valor médio

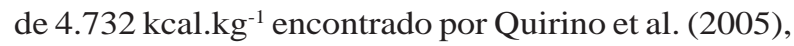
que realizaram levantamento bibliográfico dessa variável em diversas espécies utilizadas como fonte de energia.
Observou-se que a madeira de Mimosa tenuiflora obteve o maior valor médio dessa variável (4.823 kcal.kg-1), diferindo significativamente das demais. Os menores valores foram observados em Caesalpinia pyramidalis (4.442 kcal. $\left.\mathrm{kg}^{-1}\right)$, que não diferiu da madeira de Commiphora leptophloeos (4.478 kcal.kg-1).

Revista Árvore, Viçosa-MG, v.37, n.6, p.1153-1163, 2013 
Tabela 3 - Perda de massa das oito espécies em função das faixas de temperatura Table 3 - Mass loss of the eight species according to temperature ranges

\begin{tabular}{|c|c|c|c|c|c|c|c|}
\hline \multirow[b]{2}{*}{ Espécie } & \multicolumn{7}{|c|}{ Perda de massa (\%) } \\
\hline & $\begin{array}{l}30-100 \\
\left({ }^{\circ} \mathrm{C}\right)\end{array}$ & $\begin{array}{c}100-200 \\
\left({ }^{\circ} \mathrm{C}\right)\end{array}$ & $\begin{array}{c}200-300 \\
\left({ }^{\circ} \mathrm{C}\right)\end{array}$ & $\begin{array}{c}300-400 \\
\left({ }^{\circ} \mathrm{C}\right)\end{array}$ & $\begin{array}{c}400-500 \\
\left({ }^{\circ} \mathrm{C}\right)\end{array}$ & $\begin{array}{c}400-600 \\
\left({ }^{\circ} \mathrm{C}\right)\end{array}$ & $\begin{array}{c}\text { Massa residual } \\
(\%)\end{array}$ \\
\hline Mimosa tenuiflora & 10 & 1 & 18 & 38 & 6 & 4 & 23 \\
\hline Aspidosperma pyrifolium & 11 & 1 & 21 & 40 & 5 & 5 & 17 \\
\hline Croton sonderianus & 10 & 2 & 22 & 42 & 7 & 6 & 11 \\
\hline Caesalpinia pyramidalis & 9 & 2 & 22 & 37 & 6 & 3 & 21 \\
\hline Bauhinia cheilantha & 9 & 1 & 15 & 36 & 6 & 4 & 29 \\
\hline Commiphora leptophloeos & 15 & 1 & 20 & 35 & 5 & 3 & 20 \\
\hline Piptadenia stipulacea & 10 & 0 & 21 & 49 & 4 & 2 & 14 \\
\hline Combretum leprosum & 9 & 1 & 17 & 49 & 4 & 2 & 18 \\
\hline
\end{tabular}

Tabela 4 - Temperaturas de início do processo exotérmico e dos picos de máximo e mínimo das curvas de DSC das oito espécies em estudo e variação da entalpia $(\Delta \mathrm{H})$.

Table 4 - Temperatures at the beginning of the exothermic process and the maximum and minimum peaks of DSC curves of the eight species in the study and enthalpy range $(\Delta H)$.

\begin{tabular}{|c|c|c|c|c|}
\hline \multirow{2}{*}{ Espécie } & \multicolumn{3}{|c|}{ Temperatura $\left({ }^{\circ} \mathrm{C}\right)$} & \multirow{2}{*}{$\Delta \mathrm{H}\left(\mathrm{kJ} \cdot \mathrm{g}^{-1}\right)$} \\
\hline & Pico endotérmico & Início & Pico exotérmico & \\
\hline Mimosa tenuiflora & 81,44 & 332,15 & 373,96 & $-0,15$ \\
\hline Aspidosperma pyrifolium & 84,41 & 324,56 & 370,92 & $-0,06$ \\
\hline Croton sonderianus & 81,59 & 330,82 & 370,90 & 0,79 \\
\hline Caesalpinia pyramidalis & 78,23 & 327,98 & 362,51 & $-0,03$ \\
\hline Bauhinia cheilantha & 77,00 & 320,57 & 362,47 & 1,10 \\
\hline Commiphora leptophloeos & 82,09 & 324,02 & 392,96 & $-1,75$ \\
\hline Piptadenia stipulacea & 79,60 & 328,64 & 369,67 & 1,69 \\
\hline Combretum leprosum & 79,77 & 336,03 & 371,54 & 0,22 \\
\hline
\end{tabular}

Oliveira (2003) encontrou valores médios de poder calorífico inferiores aos obtidos neste trabalho, nas espécies Mimosa tenuiflora (4.482 kcal. $\mathrm{kg}^{-1}$ ), Aspidosperma pyrifolium (4.431 kcal. $\mathrm{kg}^{-1}$ ) e Croton sonderianus (4.388 kcal. $\mathrm{kg}^{-1}$ ). Resultado semelhante foi observado por Lima (1996) nas madeiras de Mimosa tenuiflora e Aspidosperma pyrifolium, nas quais foram determinados valores equivalentes a 4.150 kcal. kg-1

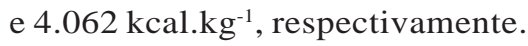

O poder calorífico é uma das principais variáveis utilizadas para seleção de espécies com melhores características para fins energéticos, uma vez que está relacionada com a quantidade de energia liberada pela madeira durante sua queima. A quantidade de calor desprendida da madeira é muito importante para se conhecer a capacidade energética de determinada espécie (SANTOS, 2010).
Devido à complexidade do comportamento da biomassa diante da pirólise, é importante destacar a influência dos componentes da biomassa. Para isso, é preciso isolar os seus principais componentes e realizar a pirólise individualmente, sem considerar as interações entre os constituintes, e somente dessa forma é possível distinguir as sobreposições de similaridades dos diferentes constituintes ao mesmo tempo (HAYKIRIACMA et al., 2010).

A segunda etapa de degradação é atribuída à pirólise dos principais constituintes da madeira, ou seja, celulose, hemiceluloses e lignina, e cada um desses componentes apresenta comportamento diferente quando exposto ao calor. Dessa forma, pode-se dizer que existe compatibilidade entre os termogramas obtidos da madeira e os obtidos dos componentes da madeira isolados (REH et al., 1986). De acordo com Yang et al. (2007),

Revista Árvore, Viçosa-MG, v.37, n.6, p.1153-1163, 2013 
as hemiceluloses iniciam sua decomposição mais facilmente, com a perda de peso, acontecendo, sobretudo, na faixa de $220^{\circ} \mathrm{C} \mathrm{a} 315^{\circ} \mathrm{C}$. Ainda segundo esses autores, a pirólise da celulose apresenta uma gama de temperatura maior, ocorrendo de $315^{\circ} \mathrm{C}$ a $400^{\circ} \mathrm{C}$, e em temperaturas superiores a $400^{\circ} \mathrm{C}$ a maior parte da celulose é degradada, apresentando baixo resíduo sólido (cerca de 6,5\%). Entre os três componentes, a lignina é de decomposição mais difícil, pois esse processo acontece lentamente desde o início da carbonização dos demais constituintes até $900^{\circ} \mathrm{C}$, com baixa taxa de perda de massa (YANG et al., 2007). É importante salientar que a lignina isolada, independentemente do procedimento utilizado, é diferente da lignina encontrada na madeira in natura (REH et al., 1986). Resultados semelhantes aos de Yang et al. (2007) foram observados por Elyounssi et al. (2012), que avaliaram madeira de Eucalyptus sp. e componentes da madeira isolados (celulose microcristalina, xilana de bétula e lignina álcali).

De acordo com Santos (2008), o carvão para uso siderúrgico deve apresentar densidade aparente acima de 0,25 g. $\mathrm{cm}^{-3}$, no entanto ressalta-se que as propriedades do carvão vegetal são bastante variáveis, pois sofrem influência da matéria-prima que lhe deu origem e do processo de carbonização, variáveis essas de difícil controle. Observou-se que, de maneira geral, as espécies estudadas apresentaram valores de densidade aparente do carvão acima de 0,25 g.cm ${ }^{-3}$, exceto a madeira de imburana (Commiphora leptophloeos), que apresentou 0,20 g.cm ${ }^{-3}$ nessa variável. O maior valor de densidade aparente foi de 0,64 g.cm ${ }^{-3}$, obtido na madeira de juremapreta (Mimosa tenuiflora), que também apresentou alta densidade básica da madeira.

De acordo com Sturion et al. (1988), maiores valores de densidade aparente do carvão vegetal conferem a ele maior resistência mecânica e maior capacidade calorífica por unidade de volume.

\section{CONCLUSÕES}

- Com base no que foi exposto, pode-se concluir que o método de TGA foi eficiente para selecionar as madeiras com maior potencial energético, uma vez que apresentou a mesma tendência de rendimento gravimétrico do método da mufla.

- As madeiras que apresentaram maior rendimento gravimétrico foram jurema-preta (Mimosa tenuiflora) e mororó (Bauhinia cheilantha), que também apresentaram os maiores valores de densidade aparente do carvão, que são variáveis importantes na escolha de espécies para fins energéticos.

- Na indicação de espécies visando ao uso como energia, é fundamental antever a qualidade da madeira mediante a avaliação das suas propriedades. Assim, para esse fim, recomenda-se o uso da madeira com maiores valores da densidade básica, poder calorífico superior e percentual de massa residual após o processo de carbonização e combustão, assim como maiores rendimentos gravimétricos em carvão vegetal.

- Os resultados deste trabalho reforçam a importância das técnicas utilizadas como ferramentas úteis para se compreender a decomposição de biomassa lenhosa diante do aquecimento em processos de conversão termoquímica.

\section{AGRADECIMENTOS}

Os autores agradecem a Embrapa Floresta, o Conselho Nacional de Desenvolvimento Científico e Tecnológico (CNPq) e a Fundação de Apoio a Pesquisa do Estado do Rio Grande do Norte (Fapern) pelo auxilio financeiro ao desenvolvimento do projeto.

\section{REFERÊNCIAS}

\section{AMERICAN SOCIETY FOR TESTING AND} MATERIALS - ASTM. Standard specification for woven wire test sieve cloth and test sieves. Philadelphia: 1974.

ARAÚJO, L. V. C.; LEITE, J. A. N.; PAES, J. B. Estimativa da produção de biomassa de um povoamento de Jurema-Preta (Mimosa tenuiflora (WILLD.) com cinco anos de idade. Biomassa \& Energia, v.1, n.4, p.347-352, 2004.

\section{ASSOCIAÇÃO BRASILEIRA DE NORMAS} TÉCNICAS - ABNT. NBR 8633. Brasília: 1984.

BERNAL, C. et al. Influência de alguns parâmetros experimentais nos resultados de análises calorimétricas diferenciais - DSC. Química Nova, v.25, n.5, p.843-855, 2002.

BRITO, J. O.; BARRICHELLO, L. E. G. Correlações entre características físicas e químicas da madeira e a produção de carvão vegetal: 1 . Densidade e teor de lignina da madeira de eucalipto. IPEF, n.14, p.9-20, 1977.

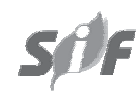

Revista Árvore, Viçosa-MG, v.37, n.6, p.1153-1163, 2013 
CARVALHO, A. G. M. Relação siringila/ guaiacila na lignina e suas implicações. 2002. 40f. Monografia (PósGraduação em Tecnologia de Celulose e Papel) Universidade Federal de Viçosa, Viçosa, MG, 2002.

CAstro, A. F. N. M. Efeito da idade e de materiais genéticos de Eucalyptus sp. na madeira e carvão vegetal. 2011. 98f. Dissertação (Mestrado em Ciencia Florestal) - Universidade Federal de Viçosa, Viçosa, MG, 2011.

CAVALHEIRO, E. T. G. et al. A influência de fatores experimentais nos resultados de análises termogravimétricas. Química Nova, v.18, n.3, p.305-308, 1995.

CONESA, J. A. et al. Analysis of different kinetic models in the dynamic pyrolisis of cellulose.

Thermochimica Acta, v.254, n.1, p.175-192, 1995.

ELYOUNSSI, K. et al. Improvement of charcoal yield by two-step pyrolysis on eucalyptus wood: A thermogravimetric study. Fuel, v.96, p.161-167, 2012.

FRANCESCHI, E.; CASCONE, I.; NOLE, D. Study of artificially degraded Woods simulating natural ageing of archaeological findings. Journal of Thermal Analysis and Calorimetry, v.92, n.1, p.319-322, 2008.

GIUlietTi, A. M. et al. Diagnóstico da vegetação nativa do Bioma Caatinga. RECIFE-PE: UFPE/RECIFE. EDITORA:

Universitária. 2003. 44p.

HAYKIRI-ACMA, H.; YAMAN, S.; KUCUKBAYRAK, S. Comparison of the thermal reactivities of isolated lignin and holocellulose during pyrolysis. Fuel Processing Technology, v.91, n.7, p.759-764, 2010.

LIMA, J. L. S. Características físico-mecânicas e energéticas de madeiras do trópico semi-árido do Nordeste do Brasil. Boletim de Pesquisa, n.63, p.1-12, 1996.

MELO, R. R. et al. Estudo da variação radial da densidade básica de sete madeiras do semiárido. Revista Científica Eletrônica de Engenharia Florestal, v.4, n.7, p.1-8, 2006.
OLIVEIRA, A. C. et al. Parâmetros de qualidade da madeira e do carvão vegetal de Eucalyptus pellita F. Muell. Scientia Forestalis, v.38, n.87, p.431-439, 2010.

OLIVEIRA, E. Características anatômicas, químicas e térmicas da madeira de três espécies de maior ocorrência no Semi-Árido Nordestino. 2003. 122f. Tese (Doutorado em Ciência Florestal) - Universidade Federal de Viçosa, Viçosa, MG, 2003.

OLIVEIRA, E. Correlações entre parâmetros de qualidade da madeira e do carvão de Eucalyptus grandis. 1988. 47f. Dissertação (Mestrado em Ciencia Florestal) - Universidade Federal de Viçosa, Viçosa, MG, 1988.

\section{OLIVEIRA, R.M. Utilização de} características de superfícies em madeiras tratadas termicamente. 2009. 127f. Tese (Doutorado em Física Aplicada) Universidade de São Paulo, São Carlos, 2009.

QUIRINO, W. F. et al. Poder calorífico da madeira e de materiais lignocelulósicos. Revista da Madeira, n.89, p.100-106, 2005.

REH, U.; KRAEPELIN, G.; LAMPRECHT, I. Use of differential scanning calorimetry for structural analysis of fungally degraded wood. Applied Environmental Microbiology, v.52, n.5, p.1101-1106, 1986.

RIEGEL, I. et al. Análise termogravimétrica da pirólise da Acacia negra (Acacia mearnsii de Willd.) cultivada no Rio Grande do Sul, Brasil. Revista Árvore, v.32, n.3, p.533-543, 2008.

SANTOS, M. A. S. Parâmetros da qualidade do carvão vegetal para uso em alto-forno. In: Fórum nacional de carvão vegetal. 2008. Belo Horizonte. Disponível em: <painelflorestal.com.br/ exibeNews.php?id=1784>. Acesso em: 31 maio, 2009.

SANTOS, R. C. Parâmetros de qualidade da madeira e do carvão vegetal de clones de eucalipto. 2010. 173f. Tese (Doutorado em Ciência e Tecnologia da Madeira) - Universidade Federal de Lavras, Lavras, 2010. 
SCHWOB, M. R. V. Perspectivas de difusão do gás natural na indústria brasileira de cerâmica vermelha. 2007. 370f. Dissertação (Mestrado em Planejamento Energético) - Universidade Federal do Rio de Janeiro, Rio de Janeiro, 2007.

SEPLAN. Plano de desenvolvimento regional do Seridó: diagnóstico. NATALRN.: Conselho de desenvolvimento sustentável do Seridó, 2004. v.1. 315p.

SILVA, G.; MATTOS, E. C.; NAKAMURA, N. M. Aplicação da calorimetria exploratória diferencial no estudo da cinética de transição $\alpha-\delta$ HMX. Química Nova, v.26, n.6, p.889-891, 2004.
SINDICER. Sindicato da Indústria Cerâmica para Construção do RN. 2012, Rio Grande do Norte. Disponível em: <sindindustria.com.br >. Acesso em: 31 de maio de 2013.

STURION, J. A.; PEREIRA, J. C. D.; CHEMIN, M. S. Qualidade da madeira de Eucalyptus viminalis para fins energéticos em função do espaçamento e da idade de corte. Boletim de Pesquisa Florestal, n.16, p.55-59, 1988.

VITAL, B. R. Métodos de determinação de densidade da madeira. Viçosa, MG: SIF, 1984. 21p. (Boletim Técnico, 1).

YANG, H. Characteristics of hemicellulose, cellulose and lignin pyrolysis. Fuel, v.86, p.1781-1788, 2007. 
